\title{
Malignant mesothelioma of the pleura: relation between histological type and clinical behaviour
}

\author{
MR LAW, MARGARET E HODSON, BE HEARD \\ From the Cardiothoracic Institute and Brompton Hospital, London
}

ABSTRACT The differing clinical behaviour of malignant mesothelioma of different cell types was studied in 115 cases of pleural mesothelioma, classified histologically into epithelial (60), sarcomatous (25), and mixed (30). Epithelial mesotheliomas were associated with clinical features characteristic of carcinomas rather than sarcomas, including spread of tumour by direct extension, large pleural effusions, contralateral pleural effusions, ascites, metastases in regional lymph nodes, and occasional response to radiotherapy. Sarcomatous mesotheliomas were associated with clinical features more characteristic of sarcomas, with more frequent distant metastases, little or no effusion, and shorter survival. Mixed tumours had features of both, large pleural effusions occurring as frequently as with epithelial tumours, but survival being almost as poor as in sarcomatous cases. Despite these differences there is evidence from published reports that epithelial, sarcomatous, and mixed mesotheliomas have a common origin from mesothelial cells or their precursor cells.

Both epithelial and sarcomatous malignant tumours of the pleura have been recognised since the nineteenth century. There was considerable debate in the early reports about whether both types of tumour should be considered primary pleural neoplasms. In 1924 Robertson' in an extensive review concluded that all primary pleural tumours were sarcomas, and that epithelial malignancies of the pleura were in reality metastases from carcinomas of the bronchus or other primary sites. In 1931 Klemperer and Rabin, ${ }^{2}$ by contrast, considered that only epithelial pleural tumours originated from mesothelial cells while sarcomatous pleural tumours arose from the connective tissue stroma of the pleura. The idea that mesothelial cells may differentiate along both epithelial and sarcomatous cell lines was suggested in 1941 when Stout and Murray ${ }^{3}$ found epithelial cells in tissue culture from a purely sarcomatous malignant mesothelioma; this finding was confirmed by later workers. ${ }^{45}$ More recently electron microscope studies have supported this idea. ${ }^{6-10}$

It is now therefore generally accepted that both varieties of tumour stem from the mesothelial cell or its precursor, and malignant mesothelioma is regarded as a single pathological entity but with a wide range of appearances encompassing epithelial,

Address for reprint requests: Dr MR Law, Brompton Hospital, Fulham Road, London SW3 6HP. sarcomatous, and mixed patterns." This cellular variability of mesotheliomas has been explained by the concept of the embryological identity of mesothelium with mesoderm, which can differentiate into several histological patterns, and the term mesodermoma has been suggested. ${ }^{12}$

In this paper we report differences in clinical behaviour associated with epithelial, sarcomatous, and mixed cell histological patterns, which have not been investigated in detail previously.

\section{Methods}

Histological material from 115 cases of malignan mesothelioma of the pleura was reviewed by one pathologist (BEH). Adequate tumour tissue was available in all cases, usually from open surgical procedures or necropsies. The tumours were classified histologically into epithelial, sarcomatous, and mixed cell types, according to the following criteria.

Epithelial type This term was applied to mesotheliomas showing some resemblance to carcinomas. particularly adenocarcinomas, with tubule formation (fig $1 a$ ). The tubules were usually lined by a simple, roughly cuboidal epithelium, supported by a benignlooking fibrous stroma, which often formed rounded nodules (fig $1 b$ ). A tubulopapillary pattern of the epithelium was common (fig 1c), but in less differen- 


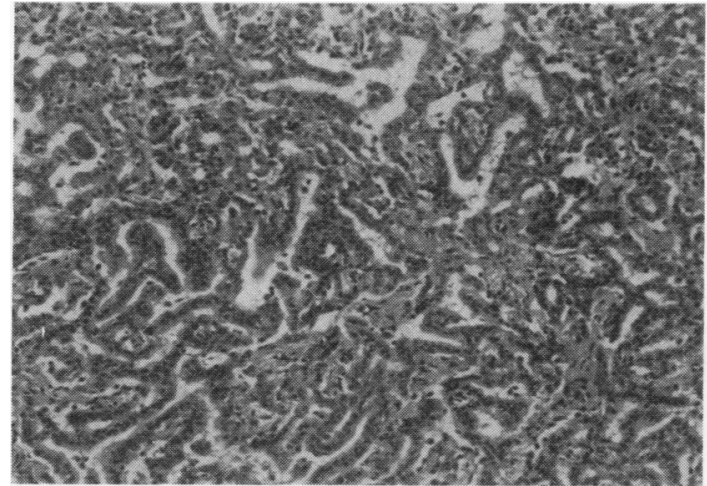

(a)

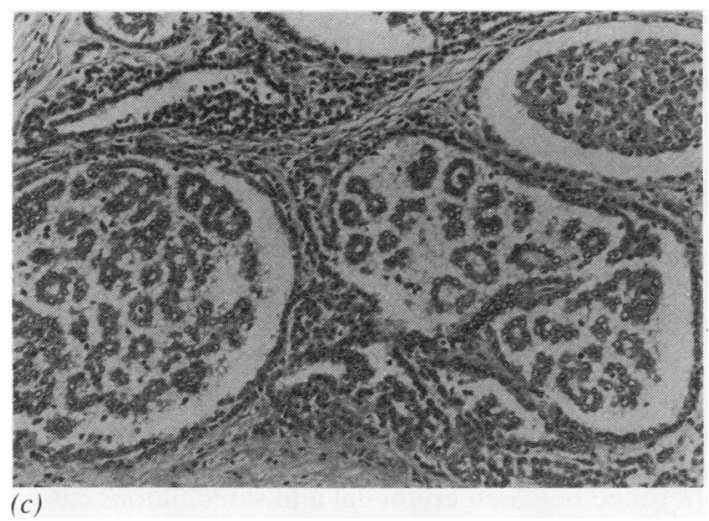

tiated tumours sheets or trabeculae of polygonal cells were found. Tumours composed of very large spheroidal cells, often with bizarre nuclei, were classified as epithelial mesotheliomas. Care was taken to try to exclude pleural deposits of adenocarcinoma from this group of epithelial mesotheliomas. Cells lining the tubules of adenocarcinomas were usually taller, and there was often mucin in the cytoplasm and lumen, confirmed by mucicarmine and diastase periodic-acid-Schiff (PAS) stains, which were used in every case. A few uncertain cases were checked by electron microscopy.

Sarcomatous type This term was applied to mesotheliomas resembling spindle-celled sarcomas and containing no epithelial element (fig $2 a$ ). Collagen and reticulin fibres could be demonstrated between individual tumour cells by special stains, and sometimes the collagen was present in very large amounts and was hyalinised. Sarcomatous mesotheliomas could generally be distinguished from benign pleural fibrosis by the greater cellularity and the presence of larger cells showing more mitoses. They lacked the storiform pattern of malignant fibrous histiocytomas. Pleural deposits of metastatic fibrosarcoma tended to

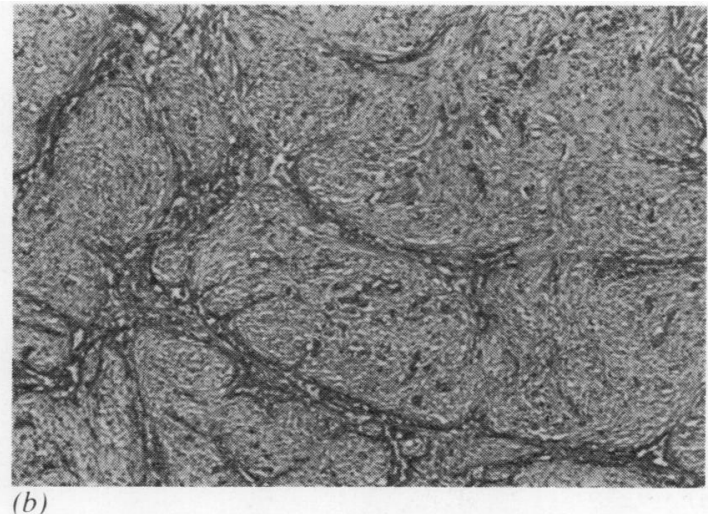

Fig 1(a) Epithelial mesothelioma with tubule formation. (b) Epithelial mesothelioma with tubules separated by rounded nodules of benign-looking fibrous stroma. (c) Epithelial mesothelioma with a tubulopapillary pattern. (Haematoxylin and eosin, $\times 50$.)

show larger, more pleomorphic tumour cells than sarcomatous mesotheliomas.

Mixed type The term mixed type was used for mesotheliomas with epithelial and sarcomatous patterns, both present in varying proportions (fig $2 b$ ). The presence of a focus of only one or two small tubules in an otherwise purely sarcomatous mesothelioma was disregarded. It was important not to mistake tubules of benign mesothelial cells in the growth for a malignant epithelial component.

The clinical features of each case, including age, symptoms and signs, presence of effusion, response to treatment, and survival, were noted. Actuarial survival curves for the three histological types were constructed, on the basis of survival from the onset of first symptoms. Postmortem findings were reviewed when available.

\section{Results}

The 115 mesotheliomas consisted of $60(52 \%)$ of epithelial type, $30(26 \%)$ of mixed cell type, and 25 $(22 \%)$ of sarcomatous type. 


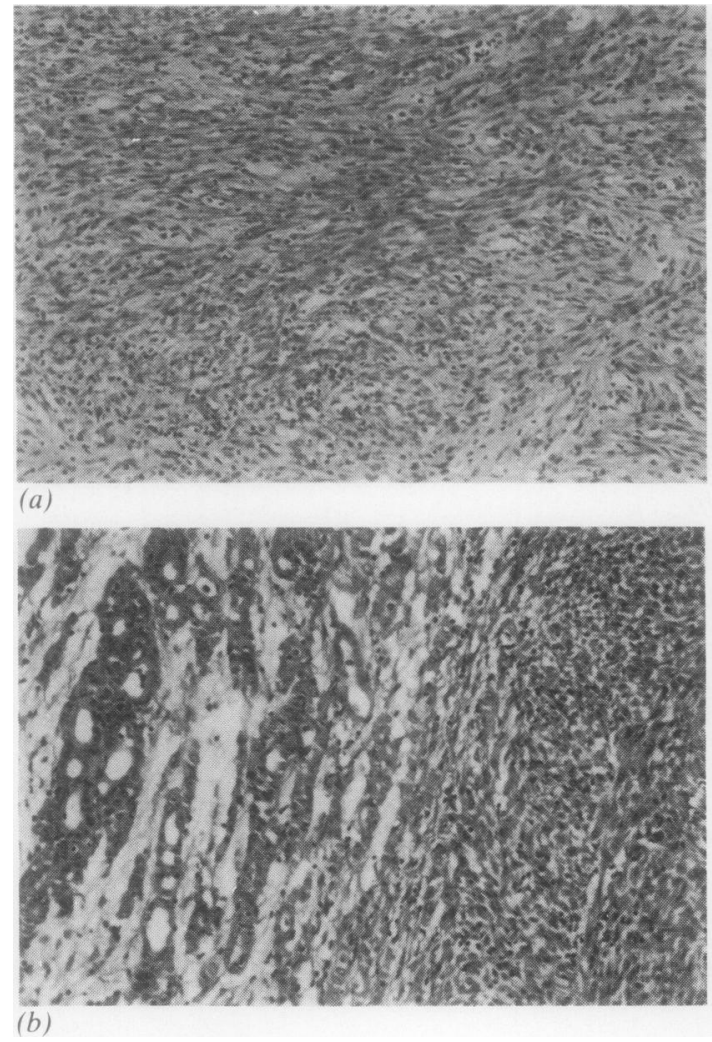

Fig 2 (a) Sarcomatous mesothelioma, composed of interlacing bands of moderately regular spindle-shaped cells. (b) Mixed mesothelioma showing rows of tubules on the left (epithelial component) and spindle-shaped cells on the right (sarcomatous component). (Haematoxylin and eosin, $\times 50$.

Certain clinical trends were similar in all three groups. The mean age at presentation was 58 years for epithelial and mixed tumours, and 59 years for sarcomatous tumours. Exposure to asbestos was documented in $48(80 \%)$ of the 60 epithelial, 23 $(77 \%)$ of the 30 mixed, and $22(88 \%)$ of the 25 sarcomatous cases. Digital clubbing was observed in four epithelial, one mixed, and three sarcomatous cases. There were no significant differences in the distributions of any of these features between the histological types.

Other features were different in the three groupsnotably the occurrence of pleural effusions. Large pleural effusions (causing dyspnoea and usually recurrent after aspiration) occurred in 71 cases and small effusions in 22 cases: in the remaining 22 cases there was no effusion. Over $70 \%$ of both epithelial and mixed tumours were associated with large effusions, whereas $84 \%$ of the sarcomatous tumours had small or no effusions (table 1 ); this difference was highly significant $\left(\mathrm{p}<0 \cdot 001 ; \chi^{2}\right.$ test $)$.

Clinically detected tumour deposits in the lymph nodes of the axilla or supraclavicular fossa were documented in $13(22 \%)$ of the 60 patients with epithelial tumours, two $(7 \%)$ of the 30 with mixed tumours, and two (8\%) of the 25 sarcomatous tumours. There were too few cases of lymph node deposits, however, for this difference to attain significance.

Forty-one patients were given radiotherapy-22 with epithelial, 10 with mixed and nine with sarcomatous tumours. Although mesotheliomas are not commonly radiosensitive there was a partial response in eight cases (relief of pain in four, regression of skin deposits in four). Seven of these tumours were epithelial and one was mixed. No patient with a sarcomatous tumour responded to radiotherapy; this difference between epithelial and sarcomatous cases was significant ( $p<0.05$; Fisher's exact test).

Survival curves for patients with epithelial, mixed, and sarcomatous tumours are shown in figure 3. During the first 18 months after the onset of symptoms the survival of patients with epithelial and mixed tumours was similar, and significantly better than that of patients with sarcomatous tumours $(p<0.01)$. Thereafter the survival of those with mixed tumours fell considerably, and epithelial tumours were associated with significantly better survival than either mixed or sarcomatous tumours up to five years ( $p<0.05$; probabilities compared on the basis of the standard errors of the curves at six-monthly intervals).

Postmortem findings were available in 60 cases.

Table 1 Occurrence of pleural effusions in tumours of different cell types

\begin{tabular}{lllll}
\hline Cell type & Total effusions & \multicolumn{2}{l}{ No $(\%)$ of patients with: } & \\
\cline { 3 - 5 } & & Large effusion & Small effusion & No effusion \\
\hline Epithelial & 60 & $46(76)^{* *}$ & $7(12)^{*}$ & $7(12)^{* *}$ \\
Mixed & 30 & $21(70)^{* *}$ & $6(20)$ NS & $3(10)^{* *}$ \\
Sarcomatous & 25 & $4(16)$ & $9(36)$ & $12(48)$ \\
Total & 115 & 71 & 22 & 22 \\
\hline
\end{tabular}

Significance of differences between sarcomatous and epithelial or mixed cell types $\left(x^{2}\right.$ test $):{ }^{*}=p<0 \cdot 05 ;{ }^{* *}=p<0 \cdot 001 ; N S=$ not significant. 


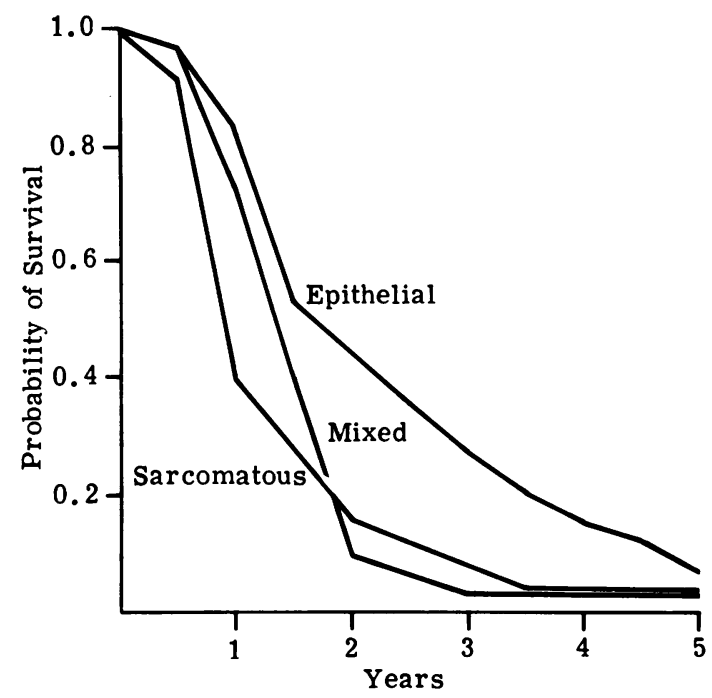

Fig 3 Survival from onset of symptoms in patients with different histological types of mesothelioma.

The number of cases with extension of tumour by direct spread to the pericardium, contralateral pleura, and peritoneum in each of the three varieties of mesothelioma is shown in table 2. Direct extension was found to be more frequent with epithelial than in mixed and sarcomatous tumours, including extension to the pericardium $(p<0.05)$ and the contralateral pleura $\left(p<0.01 ; \chi^{2}\right.$ test). Widespread extension to the pericardium, contralateral pleura, and peritoneum was far more common among epithelial tumours $(p<0 \cdot 01)$. Direct extension to the contralateral pleura was associated with large contralateral effusions during life in nine of the 19 patients with epithelial tumours but in none of the four cases of mixed or the seven cases of sarcomatous tumour in which contralateral pleural extension occurred. With peritoneal extension there was clinically apparent ascites in seven of the 13 patients with epithelial tumours and two of the eight with mixed tumours, but none of the six, with sarcomatous tumours. Both contralateral pleural effusions and ascites showed a significant association with the epithelial but not the sarcomatous cell type ( $p<0.01$; Fisher's Exact test).

The numbers of cases with distant metastases in the brain, liver, pancreas, adrenals, kidneys, and contralateral lung are also shown in table 2. Distant metastases were more commonly found at necropsy in sarcomatous than in epithelial or mixed mesotheliomas $\left(\mathrm{p}<0.025 ; \chi^{2}\right.$ test). In addition, bone metastases were diagnosed during life in three patients and confirmed by bone scan; the tumours in all three cases were sarcomatous.

\section{Discussion}

Actuarial survival curves from this series of patients with malignant mesothelioma of the pleura show significantly longer survival for those with epithelial than for those with sarcomatous mesotheliomas; the survival of patients with mixed tumours was intermediate during the first 18 months and thereafter as poor as for those with sarcomatous tumours. A longer mean survival associated with epithelial cell type has previously been reported by Elmes and Simpson. ${ }^{13}$ Ratzer et al reported that their sarcomatous mesotheliomas occurred in younger patients than their epithelial mesotheliomas, ${ }^{14}$ but in our series the cell type did not influence age at presentation. Neither did our results support a previous observation that asbestos exposure is most frequently associated with mixed tumours. ${ }^{15}$

Distant metastases were found significantly more frequently with sarcomatous mesotheliomas; this was not apparent in the smaller series of Roberts, ${ }^{16}$ but was reported by Whitwell and Rawcliffe. ${ }^{17}$ These workers and also Hourihane ${ }^{18}$ remarked that mediastinal lymph nodes were more frequently affected in the case of epithelial tumours. Davis found that in mesotheliomas induced in rats direct invasion of the surrounding tissue occurred with sarcomatous but not with epithelial varieties. ${ }^{19}$

In the present series sarcomatous mesotheliomas were not associated with frequent digital clubbing, arthralgia, or hypoglycaemia, features characteristic of benign fibrous mesothelioma of the pleura. Mixed

Table 2 Necropsy findings in tumours of different cell types

\begin{tabular}{|c|c|c|c|c|c|c|}
\hline \multirow[t]{2}{*}{ Cell type } & \multirow[t]{2}{*}{ No of necropsies } & \multicolumn{4}{|c|}{ No $(\%)$ of cases with direct extension to } & \multirow{2}{*}{$\begin{array}{l}\text { No }(\%) \text { of cases } \\
\text { with distant } \\
\text { metastasest }\end{array}$} \\
\hline & & Pericardium* & $\begin{array}{l}\text { Contralateral } \\
\text { pleura** }\end{array}$ & Peritoneum & $\begin{array}{l}\text { All three } \\
\text { surfaces ** }\end{array}$ & \\
\hline $\begin{array}{l}\text { Epithelial } \\
\text { Mixed } \\
\text { Sarcomatous }\end{array}$ & $\begin{array}{l}27 \\
15 \\
18\end{array}$ & $\begin{array}{r}19(70) \\
6(40) \\
7(39)\end{array}$ & $\begin{array}{r}19(70) \\
4(27) \\
7(39)\end{array}$ & $\begin{array}{r}13(48) \\
8(53) \\
6(33)\end{array}$ & $\begin{array}{c}12(44) \\
1(7) \\
3(17)\end{array}$ & $\begin{array}{r}10(37) \\
5(33) \\
14(78)\end{array}$ \\
\hline Total & 60 & 32 & 30 & 27 & 16 & 29 \\
\hline
\end{tabular}

${ }^{*}$ Significantly more common in epithelial than in mixed and sarcomatous tumours; $p<0 \cdot 05,{ }^{* *}=p<0 \cdot 001$. +Significantly more common in sarcomatous tumours; $p<0.025$. 
tumours tended to show certain characteristics of both cell types. They were associated with large pleural effusions almost as frequently as the epithelial tumours, but survival was almost as poor as with sarcomatous tumours. It has previously been reported that only the sarcomatous component of a mixed tumour is usually found in distant metastases. ${ }^{17}$

The histological diagnosis of mesothelioma is subject to a degree of interobserver variation, but this is lessened if adequate material is available for examination. The proportions of the different cell types in our series closely match those reported by other workers, $11315-1821$ indicating that our histological criteria are in line with theirs and that our sample is representative of mesotheliomas in general.

Epithelial mesotheliomas have certain histological characteristics that aid in their differentiation from adenocarcinomas, but sometimes the distinction cannot be made with certainty. Pleural deposits of adenocarcinomas from several primary sites, including bronchus, ovary, and large bowel, may very closely resemble epithelial mesotheliomas, particularly in small biopsy samples. ${ }^{22}$ Electron microscopy is being found useful in this differentiation. ${ }^{7-10}$ All uncertain tumours were excluded from the present study.

Similarly, pleural deposits of sarcomas may closely resemble sarcomatous mesotheliomas. We have seen two cases in which small biopsy samples suggested sarcomatous mesothelioma but in which the subsequent diagnosis, based on more tumour tissue, was pleural deposits of sarcomas from other sites - one a leiomyosarcoma of the pancreas and the other a haemangiopericytoma.

Despite the different clinical features associated with pleural tumours of epithelial and sarcomatous cell types as described above, there is good evidence for the derivation of both types of tumour from the mesothelial cell or its precursor. Evidence from tissue culture experiments ${ }^{3-5}$ has been supported by the results of electron microscope studies. ${ }^{6-10}$ Mesotheliomas induced in rats by intrapleural injection of asbestos often showed an epithelial or sarcomatous pattern only in early tumours but both patterns in more advanced tumours, ${ }^{19}$ suggesting a degree of histological mutability.

Our study shows that the clinical behaviour of epithelial mesotheliomas is more like that of carcinomas than of sarcomas, with extension along mesothelial surfaces, metastases in regional lymph nodes, formation of effusions in serous sacs, and a partial response to radiotherapy. The clinical features of sarcomatous mesotheliomas, including more frequent distant metastases and invasion of surrounding tissues, little or no effusion in serous sacs, and shorter survival, are characteristic of sarcomas in general. ${ }^{20}$
The only discrepancy in this comparison is that sarcomas usually occur in a younger age group than do carcinomas, whereas in our series the mean age was the same for both types of mesothelioma-perhaps providing further evidence for the derivation of epithelial and sarcomatous mesotheliomas from a common neoplastic mesothelial cell.

We would like to thank Professor M Turner-Warwick and other clinical colleagues of the Brompton and London Chest Hospitals whose patients we studied; Miss M Rehahn, who carried out the statistical analysis; Mr T Buckley and staff for the histological sections; and Miss S Hockley for secretarial assistance.

\section{References}

Robertson HE. Endothelioma of the pleura. $J$ Cancer Res 1924;8:317-75.

2 Klemperer P, Rabin CB. Primary neoplasms of the pleura. Arch Pathol 1931;11:385-412.

3 Stout AP, Murray MR. Localised pleural mesothelioma. Investigation of its characteristics and histogenesis by the method of tissue culture. Arch Pathol 1941;34 951-64.

4 Sano ME, Weiss E, Gault ES. Pleural mesothelioma: further evidence of its histogenesis. $J$ Thorac Surg 1950;19:783-8.

5 Alvarez-Fernandez E, Diez-Nau MD. Malignant fibrosarcomatous mesothelioma and benign pleural fibroma in tissue culture. Cancer 1979;43:1658-63.

- Bolen JW, Thorning D. Mesotheliomas: a light and electron microscopical study concerning histogenetic relationships between the epithelial and the mesenchymal variants. Am J Surg Pathol 1980;4:451-64.

${ }^{7}$ Suzuki Y, Churg J, Kannerstein M. Ultrastructure of human malignant diffuse mesothelioma. Am J Pathol 1976;85:241-51

${ }^{8}$ Echevarria RA, Arean VM. Ultrastructural evidence of secretory differentiation in a malignant pleural mesothelioma. Cancer 1968;22:323-32.

9 Wang NS. Electron microscopy in the diagnosis of pleural mesotheliomas. Cancer 1973;31:1046-54.

10 Legrand M, Pariente R. Ultrastructural study of pleural fluid in mesothelioma. Thorax 1974;29:164-71.

"McCaughey WTE. Primary tumours of the pleura. Journal of Pathology and Bacteriology 1958;76:517-29.

12 Donna A, Betta PG. Mesodermomas: a new embryological approach to primary tumours of coelomic surfaces. Histopathology 1981;5:31-44.

13 Elmes PC, Simpson MJC. The clinical aspects of mesothelioma. $Q J$ Med 1976;45:427-49.

14 Ratzer ER, Pool JL, Melamed MR. Pleural mesotheliomas-clinical experiences with 37 patients. $A J R$ 1967;99:863-80.

is McDonald AD, Magner D, Eyssen G. Primary malignant mesothelial tumours in Canada 1960-1968. A pathologic review by the Mesothelioma Panel of the Canadian Tumour Reference Centre. Cancer 1973; 31:869-76. 
16 Roberts GH. Distant visceral metastases in pleural mesothelioma. Br J Dis Chest 1976;70:246-50.

17 Whitwell F, Rawcliffe RM. Diffuse malignant pleural mesothelioma and asbestos exposure. Thorax 1971; 26:6-22.

18 Hourihane D. The pathology of mesotheliomata and an analysis of their association with asbestos exposure. Thorax 1964;19:268-78.

19 Davis JMG. The histopathology and ultrastructure of pleural mesotheliomas produced in the rat by in- jections of crocidolite asbestos. Br J Exp Pathol 1979; 60:642-52.

${ }^{20}$ Meissner WA, Diamandopoulos GT. In: Anderson WAD, Kissane JM, eds. Pathology. 7th ed. St Louis: CV Mosby Company, 1977:664-74.

21 Legha SS, Muggia FM. Pleural mesothelioma: clinical features and therapeutic implications. Ann Intern Med 1977;87:613-21.

22 Hasan FM, Nash G, Kazemi $H$. The significance of asbestos exposure in the diagnosis of mesothelioma. Am Rev Respir Dis 1977;115:761-8. 